\title{
NONCOMPACT OBJECTS AS MICROLENSES
}

\author{
A. F. ZAKHAROV \\ Institute of Theoretical and Experimental Physics, \\ B. Cheremushkinskaya, 25, 117259, Moscow \\ AND \\ M.V. SAZHIN \\ Sternberg State Astronomical Institute, \\ Universitetskij Prospekt, 13, 117234, Moscow
}

The first results of observations of microlensing have discovered a phenomenon, predicted in the papers of Byalko (1969) and Paczynsky (1986). A character of the gravitational microlens is unknown till now, although the most widespread hypothesis assumes that they are compact dark objects as brown dwarfs. Nevertheless, they could be presented by another objects, in particular, an existence of the dark objects consisting of the supersymmetrical weakly interacting particles (neutralino) has been recently discussed in the papers of Gurevich et al. We consider microlensing by a neutralino star in framework of a rough model which is rather clear and we obtain analytical expressions for results. We approximate the density of distribution mass of a neutralino star in form

$$
\rho_{N e S}(r)=\rho_{0} a_{0}^{2} / r^{2} \text {, }
$$

where $r$ is the current value of a distant from star's center, $\rho_{0}$ is mass density of a neutralino star for distance $a_{0}$ from a center, $a_{0}$ is a "radius" neutralino star. The detailed analysis of the model is presented in the papers of Zakharov \& Sazhin (1996a, 1996b). This research has been supported in part by Russian Foundation of Fundamental Research (grant N 96-0217434).

\section{References}

Zakharov, A.F. and Sazhin, M.V. (1996a) Microlensing by non-compact astronomical objects, Pis'ma Zh. Eksp. Teor. Fiz 63, pp. $894-899$.

Zakharov, A.F. and Sazhin, M.V. (1996b) Microlensing by neutralino stars, Zh. Eksp. Teor. Fiz, 110, pp. $1921-1932$. 